\title{
Exploring the Sharma-Mittal HDE models with different diagnostic tools
}

\author{
Umesh Kumar Sharma ${ }^{1 *}$, Vipin Chandra Dubey ${ }^{2 \dagger}$ \\ 1,2 Department of Mathematics, \\ Institute of Applied Sciences and Humanities, GLA University \\ Mathura-281406, Uttar Pradesh, India
}

\begin{abstract}
In this paper, we have examined the Sharma-Mittal holographic dark energy model (SMHDE) in the framework of an isotropic and spatially homogeneous flat Friedmann-Robertson-Walker(FRW) Universe by considering different values of parameter $\delta$ and $R$, where the infrared cut-off is taken care by the Hubble horizon. We examined the SMHDE model through the analysis of Statefinder hierarchy and the growth rate of perturbation. The evolutionary trajectories of the statefinder hierarchy $S_{3}^{1}, S_{3}^{2} S_{4}^{1}, S_{4}^{2}$ versus redshift z, show satisfactory behavior throughout the Universe evaluation. One promising tool for investigating the dark energy models is the composite null diagnostic(CND) $\left\{S_{3}^{1}-\epsilon\right\}$, where the evolutionary trajectories of the $S_{3}^{1}-\epsilon$ pair present different property and the departure from $\Lambda$ CDM could be well evaluated. Additionally, we investigated the dynamical analysis of the model by $\omega_{D}-\omega_{D}^{\prime}$ pair analysis.
\end{abstract}

\section{INTRODUCTION}

Various cosmological observations show that our Universe is going through accelerated expansion phase at present [1]. The concept of dark energy (DE) was used to explain this accelerated expansion of the cosmos, where DE has negative pressure [7/10]. There are basically two methods to explain the late-time acceleration of the Universe. Firstly, the dynamical dark energy model in which the matter part of the Einstein field equation can be changed. In all the theories and models, the cosmological constant model is the simplest one and was elucidated by Einstein [11 14, which gives resultant in the face of the equation of state parameter (EoS) $\omega=-1$, the most basic applicant for dark energy is the cosmological constant and it is consistent from the prospective of observations, except the coincidence and fine-tuning problem [12, 15. As an answer to the problem various dynamical dark energy models are given as an alternative like quintessence [16, 17], phantom [18, $k$-essence [19 21], tachyon [22] and Chaplygin gas [23. Secondly, by modified gravity theories, which are achieved by modifying the geometric part of Einstein field equation [24 29].

Many dark energy models have been proposed so far to explain the accelerated expansion phase of the Universe inspired by the holographic principle, which in last propagates the theory that the degree of freedom is dependent on the bounding area and not on volume 30 33. M.Li, in 2004, proposed Holographic dark energy (HDE) taking future event horizon as IR cutoff to describe the accelerated expansion scenario of the Universe [34. The holographic dark energy model has been taken into account broadly and studied in

\footnotetext{
*sharma.umesh@gla.ac.in

†vipin.dubey@gla.ac.in
}

the literature [35 39], as $\rho_{D} \propto \Lambda^{4}$, and the relation between the UV cutoff $\Lambda$, entropy $S$ and IR cutoff $L$ is $\Lambda^{3} L^{3} \leq(S)^{\frac{3}{4}}$, that shows that the combination of the entropy with the IR cut-offs gives energy density of HDE model. The declaration of $\rho_{D}$ is the focal point and is achieved by consideration of the dimensional analysis $\&$ the holographic principle instead of the inclusion of the expression of the dark energy into the Lagrangian. This is the basis for the importance of the HDE and the original Holographic DE model is dependent on Bekenstein-Hawking entropy $S=\frac{A}{4 G}$, where $A=4 \pi L^{2}$, so the density is $\rho_{D}=\frac{3 c^{2}}{8 \pi G} L^{-2}$, here $c$ is numerical constant. Three years after the HDE i. e. in 2007, Cai proposed the Agegrapic dark energy (ADE) model taking length measure as the age of the Universe [40]. Due to some confusion in the original ADE model proposed by Cai, Wei and Cai in 2008, proposed the New agegraphic dark energy (NADE) model considering conformal time as time scale [4]. The Ricci dark energy was proposed by Gao et al. 42] replacing future event horizon with Ricci scalar curvature inspired by the holographic principle.

Recently, Different entropies [43,46] have also been used to propose some new forms of dark energy model in the investigation of gravitational and the cosmological incidences. Inspired by hologrphic principle and using various system entropies, some new form of dark energy modolels were proposed, for example, the Rényi holograpic dark energy (RHDE) model [47, Tsallis holographic dark energy (THDE) model [48, Tsallis agegraphic dark energy (TADE) model [49] and Sharma-Mittal holograpic dark energy (SMHDE) model [50]. These newly proposed dark energy models were investigated by various researchers in different scenario [51-63].

As the number of dark energy models is increasing day by day, the diagnostic tools which can discrim- 
inate them are required. The statefinder hierarchy and the growth rate of linear perturbations, as null diagnostics for the $\Lambda$ CDM model, was introduced by Arabsalmani and Sahni 64 to discriminate the different dark energy models from the $\Lambda \mathrm{CDM}$ model. The statefinder hierarchy contains high derivatives of scale factor $a(t)$, model-independent and is a geometrical diagnostic [65]. Previously to check scale-independent consistency between the structure growth and the expansion history, the growth rate of the structure was used in 66 68. It can be combined with the statefinder hierarchy or act as a cosmic growth history diagnostic to serve on a composite diagnostic. Four holographic DE models were discriminated against by these two diagnostics in 65. In 68 6 76 these diagnostics were considered. Recently, the discrimination between THDE models $\Lambda$ CDM model investigated by one of the authors through statefinder hierarchy in the nonflat Universe considering apparent horizon as IR cutoff [77. The $\omega_{D}-\omega_{D}^{\prime}$ analysis [78, can also be utilized to recognize the difference in dark energy models, which is based on the behavior of EoS for the dark energy models.

In this work, we have explored the newly proposed Sharma-Mittal Holographic Dark Energy (SMHDE) model through the diagnostic tools described above in the flat FRW Universe by taking the Hubble horizon as an infrared cutoff, which has not been explored earlier. Also, we have examined the deviation of the SMHDE model from $\Lambda$ CDM using these diagnostic tools. This paper is structured as follows; In Section II, we briefly visit the Sharma-Mittal holographic dark energy. Section III is dedicated to discussing the flat FRW cosmological model. Section IV is divided into three subsections A, B and $\mathrm{C}$ for the methods of the statefinder hierarchy diagnostic and growth rate of perturbations and diagnostic by the $\omega_{D}-\omega_{D}^{\prime}$ analysis. Finally, in the last section, we have given inferences.

\section{SHARMA-MITTAL HDE MODEL}

Recently, inspired by holographic principle and using generalized entropy measure, proposed by Sharma-Mittal [46], a new form of holographic dark energy model is proposed in 50, called Sharma-Mittal holographic dark energy.

By combining the Tsallis and Rényi entropies [44, 45], which are two well-known generalized one-parametric entropy measures, with each other, a two-parametric entropy, which was introduced by Sharma-Mittal, is defined in 46 ]

$$
S_{S M}=\frac{\left(\frac{A \delta}{4}+1\right)^{R / \delta}-1}{R},
$$

where $A=4 \pi L^{2}$ and the IR cutoff is L. Where two free parameters are $\mathrm{R}$ and $\delta$. By considering proper limits of R, Rényi and Tsallis entropies can be recovered from it. The Sharma-Mittal entropy becomes Rényi entropy in the limit $R \rightarrow 0$, and in limit $R \rightarrow 1-\delta$, it becomes Tsallis entropy. The energy density is obtained when the UV cutoff and IR cutoff are taken into consideration as was suggested by Cohen et al. [79].

$$
\rho_{D} \propto \frac{S_{S M}}{L^{4}} \Longrightarrow \rho_{D}=\frac{3 c^{2} S_{S M}}{8 \pi L^{4}},
$$

Considering Hubble horizon cut-off $L=\frac{1}{H}$, when we take the aforementioned equation into consideration then the energy density of Sharma-Mittal HDE model [50] is

$$
\rho_{D}=\frac{\left(3 c^{2} H^{4}\right)\left(\left(\frac{\pi \delta}{H^{2}}+1\right)^{R / \delta}-1\right)}{8 \pi R},
$$

where $c^{2}$ is a numerical constant as usual.

\section{THE COSMOLOGICAL MODEL}

For the flat FRW Universe, the metric is given as :

$$
d s^{2}=-d t^{2}+a^{2}(t)\left(d r^{2}+r^{2} d \Omega^{2}\right)
$$

In a flat FRW Universe, the first Friedmann equation, involving dark matter and SMHDE is defined as :

$$
H^{2}=\frac{1}{3}(8 \pi G)\left(\rho_{D}+\rho_{m}\right)
$$

where $\rho_{D}$ and $\rho_{m}$ represent the energy density of SMHDE and matter, respectively. The energy density parameter of SMHDE and pressureless matter using the fractional energy densities, can be given as

$$
\Omega_{m}=\frac{8 \pi \rho_{m} G}{3 H^{2}}, \quad \Omega_{D}=\frac{8 \pi \rho_{D} G}{3 H^{2}},
$$

Now Eq. (5) with help of Eq. (6) can be written as:

$$
1=\Omega_{D}+\Omega_{m}
$$

The conservation law for matter and SMHDE are given as :

$$
\begin{gathered}
\dot{\rho}_{m}+3 H \rho_{m}=0, \\
\dot{\rho}_{D}+3 H\left(\rho_{D}+p_{D}\right)=0 .
\end{gathered}
$$

in which $\omega_{D}=p_{D} / \rho_{D}$ represents the SMHDE EoS parameter. Now, using differential with time of Eq. (5) in Eq. (8), and Eqs. (9) combined the result with the Eq. (7), we get 


$$
\begin{aligned}
& \frac{\dot{H}}{H^{2}}=-\left(3\left(1-\Omega_{D}\right)\left(\pi \delta+H^{2}\right)\right) \times \\
& \frac{1}{2\left(\pi c^{2} H^{2}\left(\frac{\pi \delta}{H^{2}}+1\right)^{R / \delta}+\pi \delta-2 \pi \delta \Omega_{D}-2 H^{2} \Omega_{D}+H^{2}\right)}(10)
\end{aligned}
$$

By Eq. 10, The deceleration parameter $q$ is found as

$$
\begin{gathered}
q=-1-\frac{\dot{H}}{H^{2}} \\
=-1+\frac{3\left(\Omega_{D}-1\right)\left(\pi \delta+H^{2}\right)}{2\left(2 \Omega_{D}-1\right)\left(\pi \delta+H^{2}\right)-2 \pi c^{2} H^{2}\left(\frac{\pi \delta}{H^{2}}+1\right)^{R / \delta}}(11)
\end{gathered}
$$

Now, taking the differential with respect to time of Eq. (3), we get

$$
\dot{\rho_{D}}=\frac{4 \rho_{D} \dot{H}}{H}-\frac{3}{4} c^{2} H \dot{H}\left(\frac{\pi \delta}{H^{2}}+1\right)^{\frac{R}{\delta}-1}
$$

Now by using the Eqs. (12) with Eqs. (9) and 10, we gets expression for EoS parameter as:

$$
\begin{aligned}
& \omega_{D}=\Omega_{D}^{-1}\left(1-\left(\Omega_{D}-1\right)\left(\pi \delta+H^{2}\right) \times\right. \\
& \left.\frac{1}{\pi c^{2} H^{2}\left(\frac{\pi \delta}{H^{2}}+1\right)^{R / \delta}-\left(2 \Omega_{D}-1\right)\left(\pi \delta+H^{2}\right)}\right)
\end{aligned}
$$

Also, taking the time differential of the energy density parameter $\Omega_{D}$ with Eqs. (10) and $\sqrt{12}$, we find

$$
\begin{gathered}
\Omega_{D}^{\prime}=-\left(3 ( \Omega _ { D } - 1 ) \left(\pi\left(-c^{2}\right) H^{2}\left(\frac{\pi \delta}{H^{2}}+1\right)^{R / \delta}\right.\right. \\
\left.+\pi \delta \Omega_{D}+H^{2} \Omega_{D}\right) \times\left(\pi\left(-c^{2}\right) H^{2}\left(\frac{\pi \delta}{H^{2}}+1\right)^{R / \delta}-\right. \\
\left.\pi \delta+2 \pi \delta \Omega_{D}+2 H^{2} \Omega_{D}-H^{2}\right)^{-1}
\end{gathered}
$$

where the dot is the derivative while taking time into consideration and prime lets us obtain the derivative with respect to $\ln$ a.

\section{THE METHODS OF DIAGNOSTIC}

In this work, we used three diagnostic tools, statefinder hierarchy, the growth rate of perturbations and $\omega_{D}-\omega_{D}^{\prime}$ pair. We shall explore the SMHDE model to discriminate from the $\Lambda \mathrm{CDM}$ model with the help of three diagnostic tools in this section.

\section{A. The Statefinder Hierarchy diagnostic}

Here, statefinder hierarchy diagnostic will be reviewed and then the growth rate of structure of the SMHDE model will be described. The Taylor expansion of the
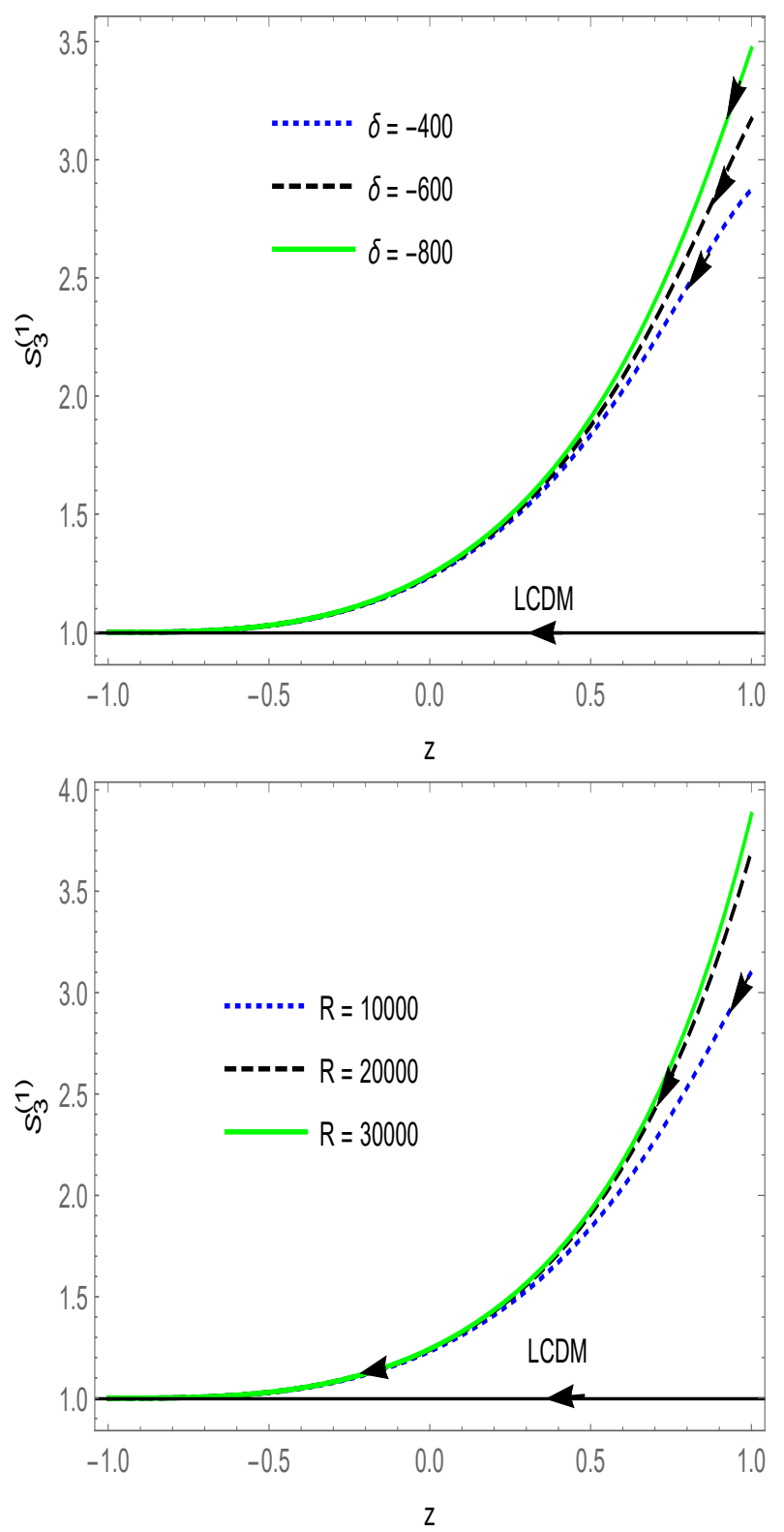

FIG. 1: Graph of $S_{3}^{(1)}$ versus redshift z, for non- interacting SMHDE with Hbbble radius as the IR cutoff. Here, $H(z=$ $0)=67, \Omega_{m}(z=0)=0.26, R=10000$ and different values of $\delta$ (upper panel) and $H(z=0)=67, \Omega_{m}(z=0)=0.26$, $\delta=-600$ and different values of $\mathrm{R}$ (below panel).

scale factor $\frac{a(t)}{a_{0}}=\frac{1}{z+1}$, around the present epoch $t_{0}$ is given as:

$$
\frac{a(t)}{a_{0}}=\sum_{n=1}^{\infty} \frac{A_{n}\left(t_{0}\right)}{n !}\left[H_{0}\left(t-t_{0}\right)\right]^{n}
$$

Where $A_{n}=\frac{a^{n}}{a H^{n}}, a^{n}$ is the $n^{t h}$ derivative of the scale factor a verses cosmic time $\mathrm{t}$ and $\mathrm{n} \in \mathrm{N}$. The statefinder 

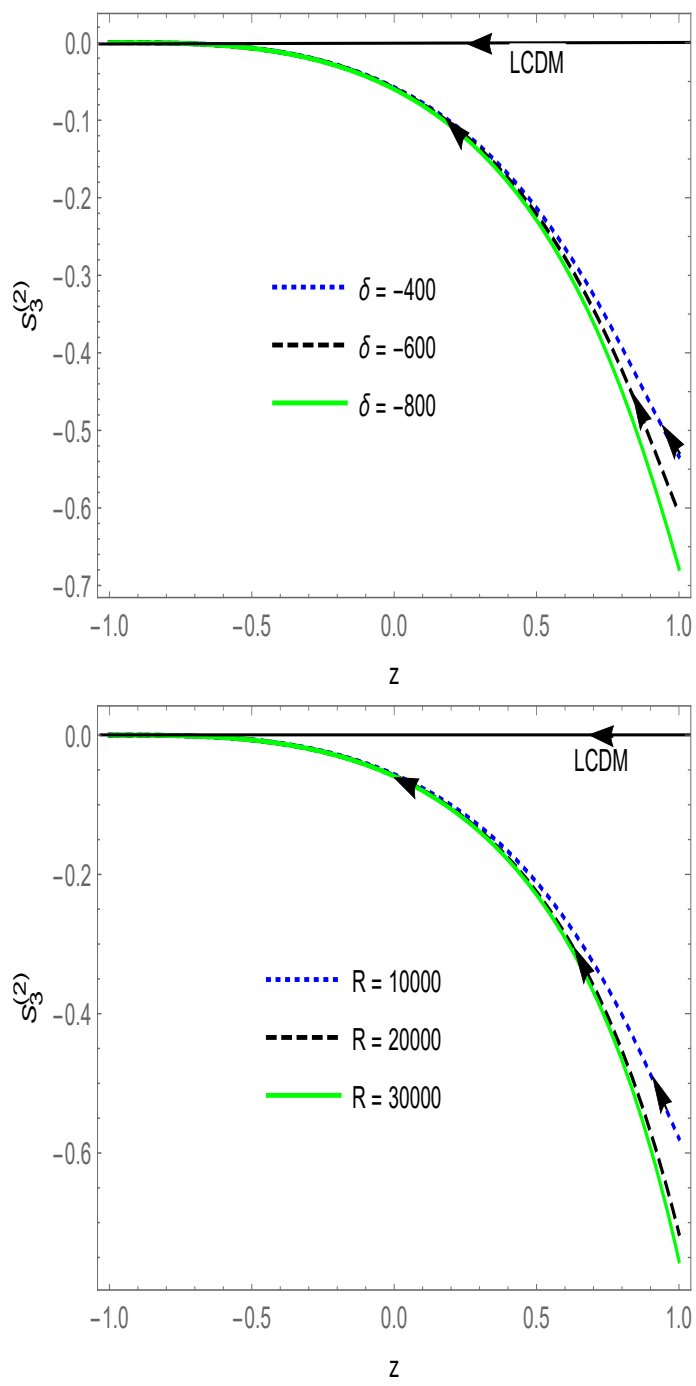

FIG. 2: Graph of $S_{3}^{(2)}$ versus redshift z, for non- interacting SMHDE with Hbbble radius as the IR cutoff. Here, $H(z=$ $0)=67, \Omega_{m}(z=0)=0.26, R=10000$ and different values of $\delta$ (upper panel) and $H(z=0)=67, \Omega_{m}(z=0)=0.26$, $\delta=-600$ and different values of $\mathrm{R}$ (below panel).

hierarchy $S_{n}$ is defined as follows [64]:

$$
S_{2}=A_{2}+\frac{3 \Omega_{m}}{2}, S_{3}=A_{3} \quad \text { and } \quad S_{4}=A_{4}+\frac{9 \Omega_{m}}{2}
$$

Aforementioned gives the diagnostics for the model $(\Lambda \mathrm{CDM})$ with $n \geq 3$, i.e., $S_{n} \mid \Lambda \mathrm{CDM}=1$. Hence by the use of $\Omega_{m}=\frac{2(q+1)}{3}$ the statefinder hierarchy $S_{3}^{(1)}$, $S_{4}^{(1)}$ can be written as:

$$
S_{3}^{(1)}=A_{3}, \quad \text { and } \quad S_{4}^{(1)}=A_{4}+3(q+1),
$$

For $\Lambda$ CDM model, $S_{n}^{(1)}=1$. In 80, it gives a path for
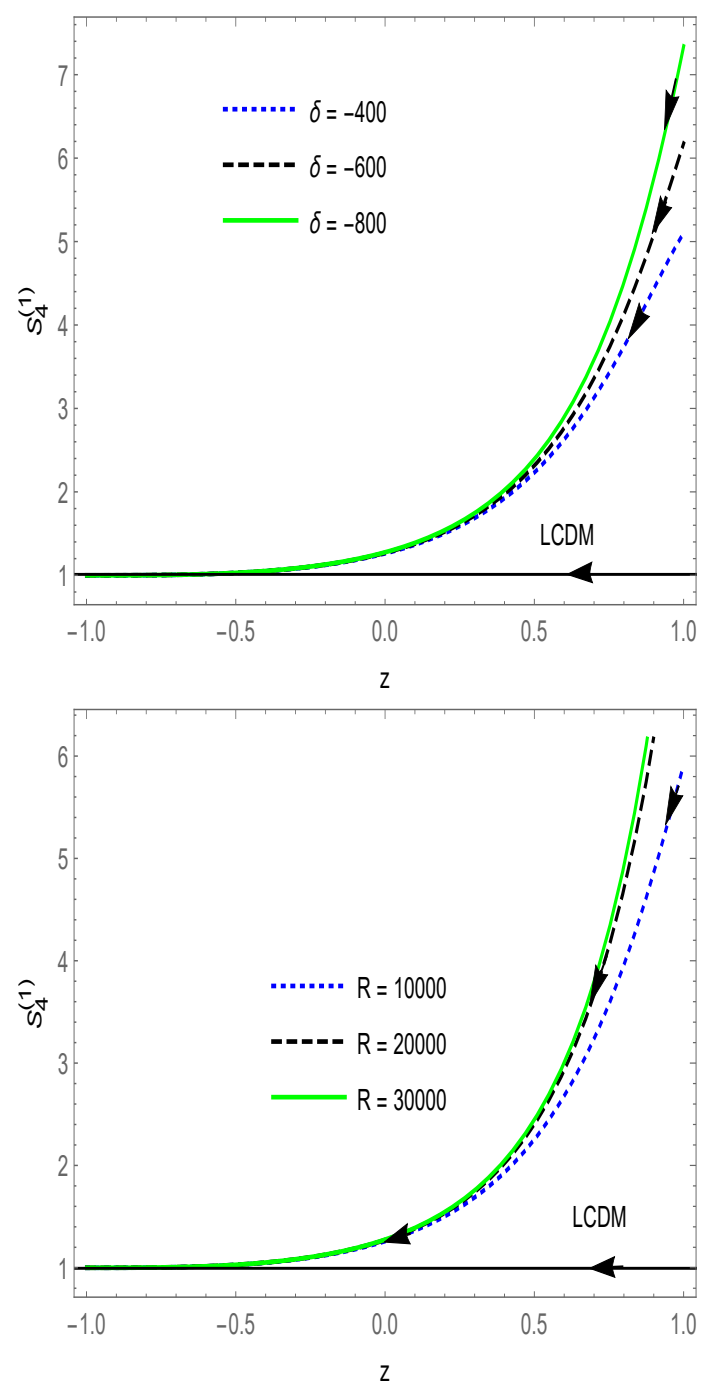

FIG. 3: Graph of $S_{4}^{(1)}$ versus redshift z, for non- interacting SMHDE with Hbbble radius as the IR cutoff. Here, $H(z=$ $0)=67, \Omega_{m}(z=0)=0.26, R=10000$ and different values of $\delta$ (upper panel) and $H(z=0)=67, \Omega_{m}(z=0)=0.26$ $\delta=-600$ and different values of $\mathrm{R}$ (below panel).

construction of second Statefinder $S_{3}^{(1)}=S_{3}$ namely

$$
S_{3}^{(2)}=\frac{S_{3}^{(1)}-1}{3\left(q-\frac{1}{2}\right)}
$$

In concordance cosmology $S_{3}^{(1)}=1$ while $S_{3}^{(2)=0}$. Hence, $\left\{S_{3}^{(1)}, S_{3}^{(2)}\right\}=\{1,0\}$ gives a model independent means for forming a distinction between the dark energy models from the cosmological constant [80. Eq. 18] gives the second member of the Statefinder hierarchy

$$
S_{n}^{(2)}=\frac{S_{n}^{(1)}-1}{\alpha\left(q-\frac{1}{2}\right)},
$$



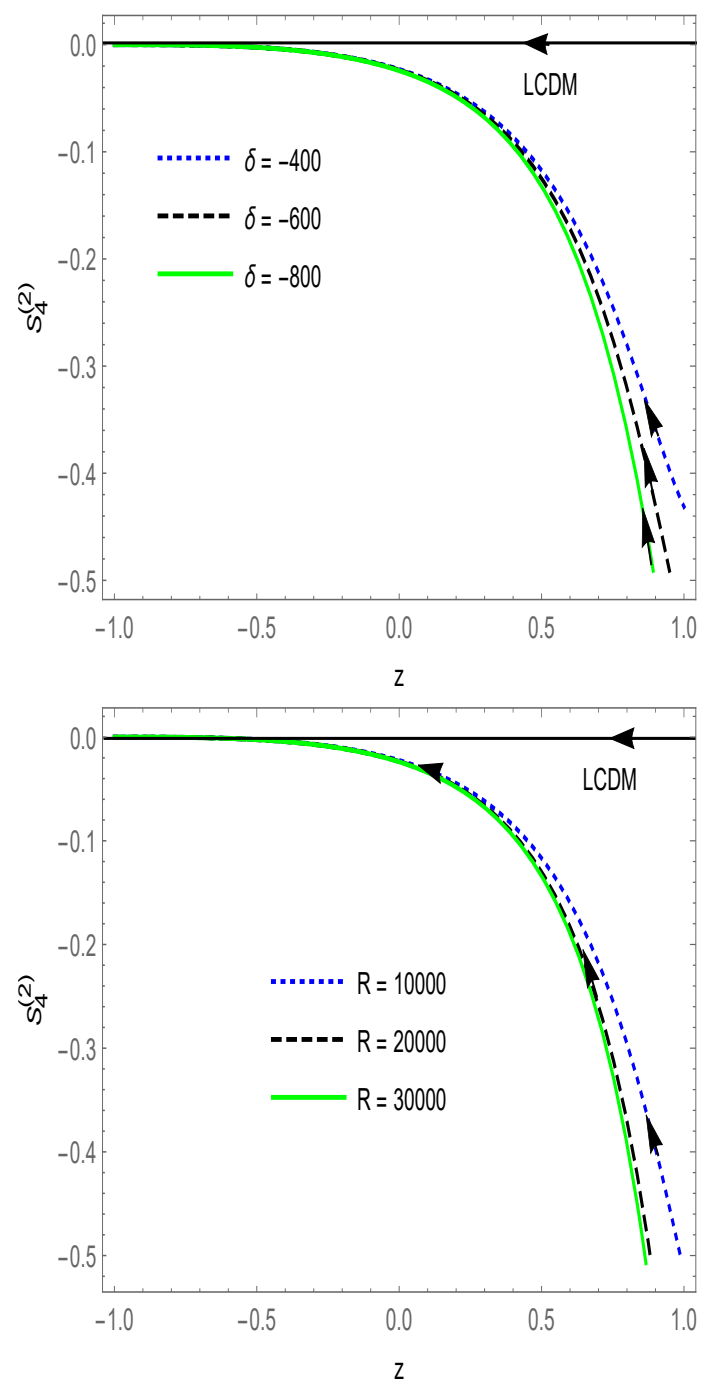

FIG. 4: Graph of $S_{4}^{(2)}$ versus redshift z, for non- interacting SMHDE with Hbbble radius as the IR cutoff. Here, $H(z=$ $0)=67, \Omega_{m}(z=0)=0.26, R=10000$ and different values of $\delta$ (upper panel) and $H(z=0)=67, \Omega_{m}(z=0)=0.26$, $\delta=-600$ and different values of $\mathrm{R}$ (below panel).

where $\alpha$ is an arbitrary constant. In concordance cos$\operatorname{mology} S_{n}^{(2)}=0$ and

$$
\left\{S_{n}^{(1)}, S_{n}^{(2)}\right\}=\{1,0\}
$$

Some of degeneracies in $S_{n}^{(1)}$ can be removed by using the second statefinder $S_{n}^{(2)}$. For the dark energy model, we have

$$
\begin{gathered}
S_{3}^{(1)}=\frac{1}{2}\left(9 \omega_{D}\right)\left(\omega_{D}+1\right) \Omega_{D}+1 \\
S_{3}^{(2)}=\omega_{D}+1 \\
S_{4}^{(1)}=-\frac{1}{4}\left(27 \omega_{D}^{2}\right)\left(\omega_{D}+1\right) \Omega^{2}{ }_{D-}^{-}
\end{gathered}
$$

$$
\begin{gathered}
\frac{1}{2}\left(27 \omega_{D}\right)\left(\omega_{D}+1\right)\left(\omega_{D}+\frac{7}{6}\right) \Omega_{D}+1 \\
S_{4}^{(2)}=-\frac{1}{2} \omega_{D}\left(\omega_{D}+1\right) \Omega_{D}-\left(\omega_{D}+1\right)\left(\omega_{D}+\frac{7}{6}\right)
\end{gathered}
$$

where $S_{4}^{(2)}=\frac{S_{4}^{(1)}-1}{9\left(q-\frac{1}{2}\right)}$ and $q-\frac{1}{2}=\frac{1}{2}\left(3 \omega_{D}\right) \Omega_{D}$. As we demonstrate in figures 1, 2, 3, 4 the Statefinder hierarchy $\left\{S_{n}^{(1)}, S_{n}^{(2)}\right\}$ give us a nice way to differenciating dynamical dark energy models from $\Lambda$ CDM model.

Fig. 1, shows the evolutionary trajectories of $S_{3}^{(1)}(z)$ for the SMHDE model by considering different values of $\delta$ (upper panel) and $R$ (below panel). Hence, we investigate two cases. The first is varying $\delta$ with a fixed $R$ (upper panels), the second is varying $R$ and a fixed $\delta$ (below panel). For the evolution of $S_{3}^{(1)}(z)$ in the SMHDE with varying $R$, the separation of curvilinear shape is not distinct from the SMHDE with varying $\delta$. In the case of varying $\delta$ or $R$ in SMHDE (upper panel or below panel), the curves which are of $S_{3}^{(1)}(z)$ have the trajectories on the line of being similar and the trend, which is being followed by of curves $S_{3}^{(1)}(z)$ is monotonically decreasing at the high-redshift region and then followed by close degeneration together into $\Lambda \mathrm{CDM} S_{3}^{(1)}=1$, at low-redshift region. This shows that different values of $\delta$ or $R$ have quantitative impacts on the $S_{3}^{(1)}(z)$. Although, in both panels, the curves discriminate well from $\Lambda \mathrm{CDM}$ in the high-redshift region but highly degenerate in the low-redshift region.

Fig. 2, shows the evolutionary trajectories of $S_{3}^{(2)}(z)$ for the SMHDE model by considering different values of $\delta$ (upper panel) and $R$ (below panel). For the evolution of $S_{3}^{(2)}(z)$ in the SMHDE with varying R, the differentiation of curvilinear shape is not distinct from the SMHDE with varying $\delta$. In the case of varying $\delta$ or $R$ in the SMHDE (upper panel or below panel), the curves which are of $S_{3}^{(2)}(z)$ have the trajectories on the line of being similar and the trend, which is being followed by of curves $S_{3}^{(2)}(z)$ is monotonically increasing at the high-redshift region and then followed by close degeneracy together with $\Lambda \mathrm{CDM} S_{3}^{(2)}=0$, at low-redshift region. This shows that different values of $\delta$ or R have quantitative impacts on the $S_{3}^{(2)}(z)$. In Fig. 3, we give the graph for $S_{4}^{(1)}$ evolution versus $z$ i.e. redshift for the SMHDE model by considering different values of $\delta$ (upper panel) and $\mathrm{R}$ (below panel). We can say that the evolutionary trajectories of $S_{4}^{(1)}(z)$ are like that of $S_{3}^{(1)}(z)$. Quantitative impacts the SMHDE model are found by adopting different values of $\delta$ and $R$. 
In Fig. 4, we give the graph for $S_{4}^{(2)}$ evolution versus $z$ i.e. redshift for the SMHDE model by considering different values of $\delta$ (upper panel) and $\mathrm{R}$ (below panel). We can say that the evolutionary trajectories of $S_{4}^{(2)}$ are like that of $S_{3}^{(2)}(z)$. Quantitative impacts on the SMHDE model are found by adopting different values of $\delta$ and $R$ and this is endorsed by the figures.

Therefore, in all the plots i.e. Fig. 1-4, there is a drawback that the curves are highly degenerate in the high-redshift region and superposing that of $\Lambda \mathrm{CDM}$ in the low-redshift region. It means that the single geometric diagnostic is not sufficient. It will be better to combine with the growth rate of perturbations, as CND for getting more clear discrimination.

\section{B. Growth rate of perturbations}

The fractional growth parameter $\epsilon(z)[66$, 67] is determined as

$$
\epsilon(z)=\frac{f(z)}{f_{\Lambda \mathrm{CDM}}(z)}
$$

Here $f(z)=\frac{d \log \delta}{d \log a}$ is the growth rate of structure. Here, $\delta=\frac{\delta \rho_{m}}{\rho_{m}}$, with $\delta \rho_{m}$ and $\rho_{m}$ being the the density perturbation and energy density of matter (including CDM and baryons), respectively. If the perturbation is in the linear fashion and without any interaction between DM and $\mathrm{DE}$, then we can say that the equation of perturbation at late times can be:

$$
\ddot{\delta}+2 \dot{\delta} H=4 \pi \delta G \rho_{m}
$$

Here, Newton's gravitational constant is represented by $G$. So, the approx growth rate of linear density perturbation can be reflected by [81:

$$
\begin{gathered}
f(z) \simeq \Omega_{m}(z)^{\gamma} \\
\gamma(z)=\frac{\left(3\left(1-\omega_{D}\right)\left(1-\frac{3 \omega_{D}}{2}\right)\right)\left(1-\Omega_{m}(z)\right)}{125\left(1-\frac{6 \omega_{D}}{5}\right)^{3}}+ \\
\frac{3}{5-\frac{\omega_{D}}{1-\omega_{D}}}
\end{gathered}
$$

where $\Omega_{m}(z)=\frac{\rho_{m}(z)}{3 H(z)^{2} M_{p}^{2}}$, the fractional density of matter, $\Omega$ is constant or varies slowly with time. $\epsilon(z)$ $=1$ and $\gamma \simeq 0.55$ are the values for the $\Lambda$ CDM model [81, 82. For other models $\epsilon(z)$ exhibits differences from $\Lambda \mathrm{CDM}$ which would be the possible reason for its use as a diagnostic. By applying the composite null diagnostic $C N D \equiv\left\{S_{n}, \epsilon\right\}$ where $\left\{S_{n}, \epsilon\right\}=\{1,1\}$ for $\Lambda$ CDM, we can make use of both matter perturbational as well as geometrical information of cosmic evolution. While, we
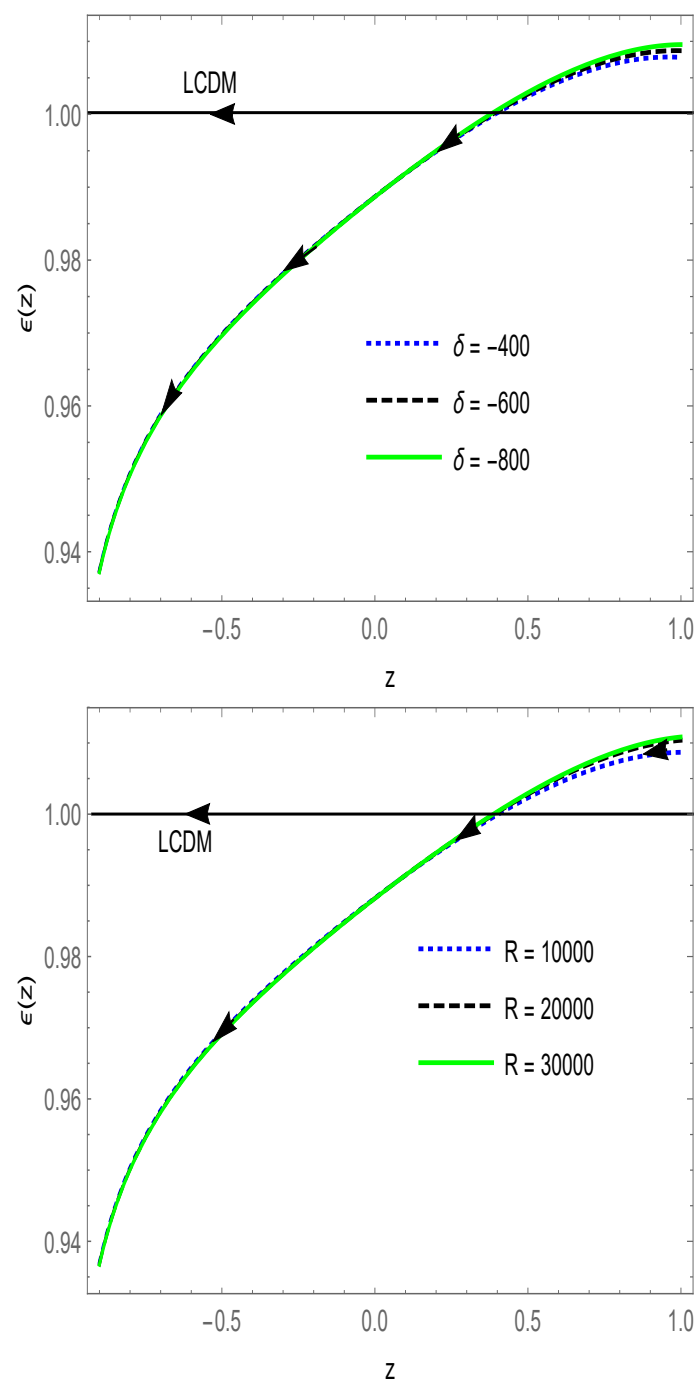

FIG. 5: Graph of $\epsilon(z)$ versus redshift z, for non- interacting SMHDE with Hbbble radius as the IR cutoff. Here, $H(z=$ $0)=67, \Omega_{m}(z=0)=0.26, R=10000$ and different values of $\delta$ (upper panel) and $H(z=0)=67, \Omega_{m}(z=0)=0.26$, $\delta=-600$ and different values of $\mathrm{R}$ (below panel).

can analyze and present only one-side information of cosmic evolution by using one single diagnostic tool.

For the diagnose of diverse theoretical DE models, having CND pairs, $\left\{S_{3}^{(1)}, \epsilon\right\}$ and $\left\{S_{4}^{(1)}, \epsilon\right\}$, the evolution of the fractional growth parameter $\epsilon(z)$ is analysed. Fig. 5 is the evolutionary trajectories of $\epsilon(z)$ versus redshift $z$ for a spatially homogeneous and an isotropic flat FRW Universe of SMHDE model by considering different values of $\delta$ (upper panel) and $R$ (below panel). For the evolution of $\epsilon(z)$ in the SMHDE with varying $R$, the differentiation of curvilinear shape is not distinct from the SMHDE with varying $\delta$. We can say that the evolutionary trajectories of $\epsilon(z)$ have similar evolutionary trajectories. It is clear from Fig. 5, that the 

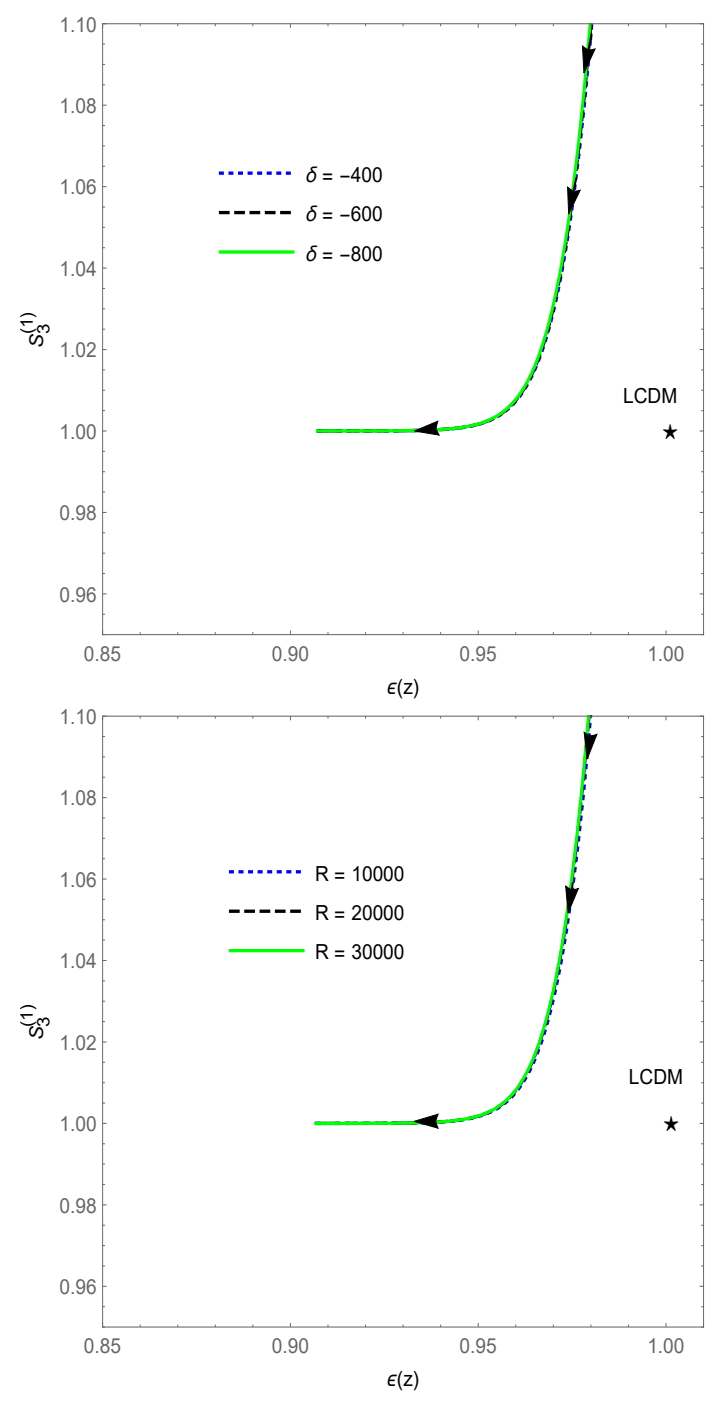

FIG. 6: Graph of Graph of $S_{3}^{(1)}$ versus $\epsilon(z)$, for non- interacting SMHDE with Hbbble radius as the IR cutoff. Here, $H(z=0)=67, \Omega_{m}(z=0)=0.26, R=10000$ and different values of $\delta$ (upper panel) and $H(z=0)=67$, $\Omega_{m}(z=0)=0.26, \delta=-600$ and different values of $\mathrm{R}$ (below panel).

evolutionary trajectories of $\epsilon(z)$ comes closer to 1 from past to future.

The evolutionary trajectories of $\left\{S_{3}^{(1)}, \epsilon\right\}$ of SMHDE model are plotted in Fig. 6 for a spatially homogeneous and an isotropic flat FRW Universe of SMHDE model by considering different values of $\delta$ (upper panel) and $R$ (below panel). The fixed point $(1,1)$ in this figure presented by by star symbol denotes the $\Lambda$ CDM. The trend of curves $\left\{S_{3}^{(1)}, \epsilon\right\}$ is monotonically decreasing from the high-redshift region to low red-shift region for the SMHDE model. This figure clearly detpicts the deviation from $\Lambda \mathrm{CDM}$ model $\left\{S_{3}^{(1)}=1, \epsilon=1\right\}$ for all
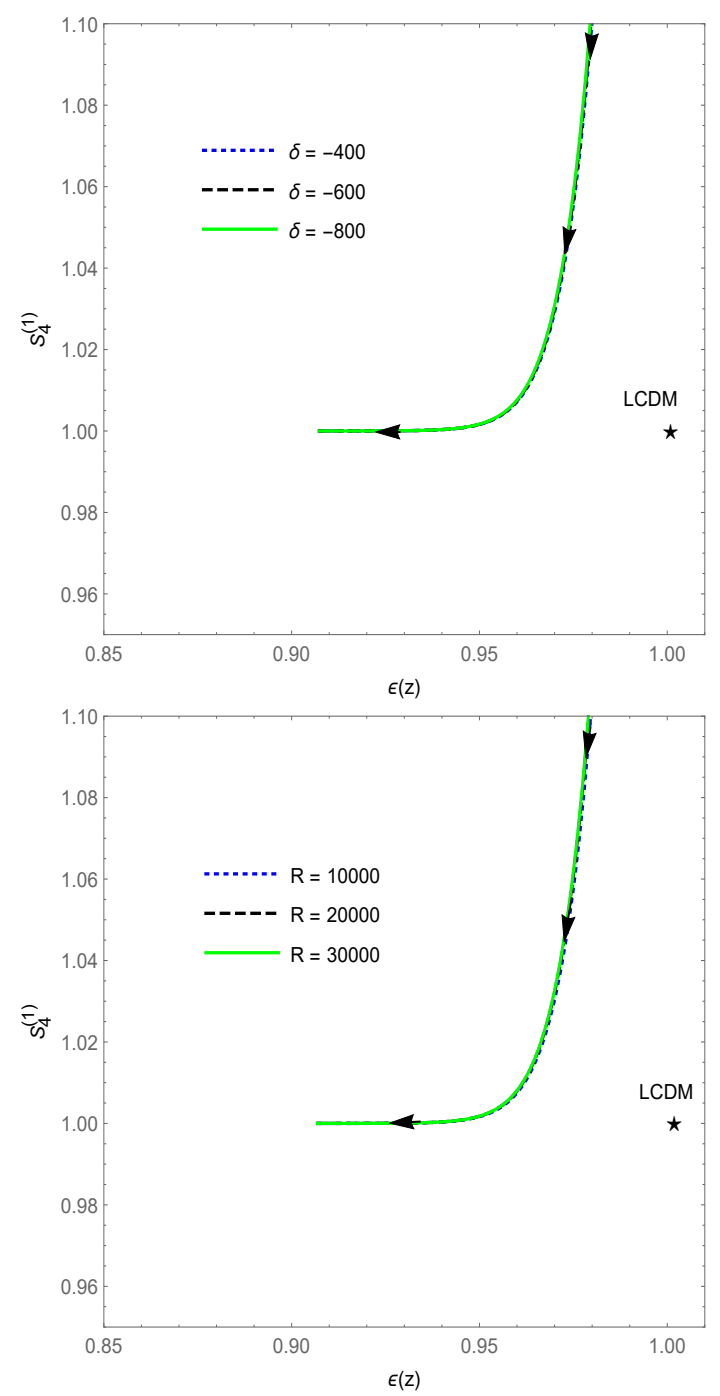

FIG. 7: Graph of Graph of $S_{4}^{(1)}$ versus $\epsilon(z)$, for non- interacting SMHDE with Hbbble radius as the IR cutoff. Here, $H(z=0)=67, \Omega_{m}(z=0)=0.26, R=10000$ and different values of $\delta$ (upper panel) and $H(z=0)=67$, $\Omega_{m}(z=0)=0.26, \delta=-600$ and different values of $\mathrm{R}$ (below panel).

values of $\delta$ and $R$ of the SMHDE model.

Fig. 7 is the the evolutionary trajectories of the CND pair $\left\{S_{4}^{(1)}, \epsilon\right\}$ for the SMHDE model by considering different values of $\delta$ (upper panel) and R (below panel). The evolutionary trajectories of $\left\{S_{4}^{(1)}, \epsilon\right\}$ shows similar characteristic as the curves of $\left\{S_{3}^{(1)}, \epsilon\right\}$. These results shows that adopting different values of $\delta$ and $\mathrm{R}$ has quantitative impacts and the deviation from $\Lambda \mathrm{CDM}$ can be seen in this figure. 

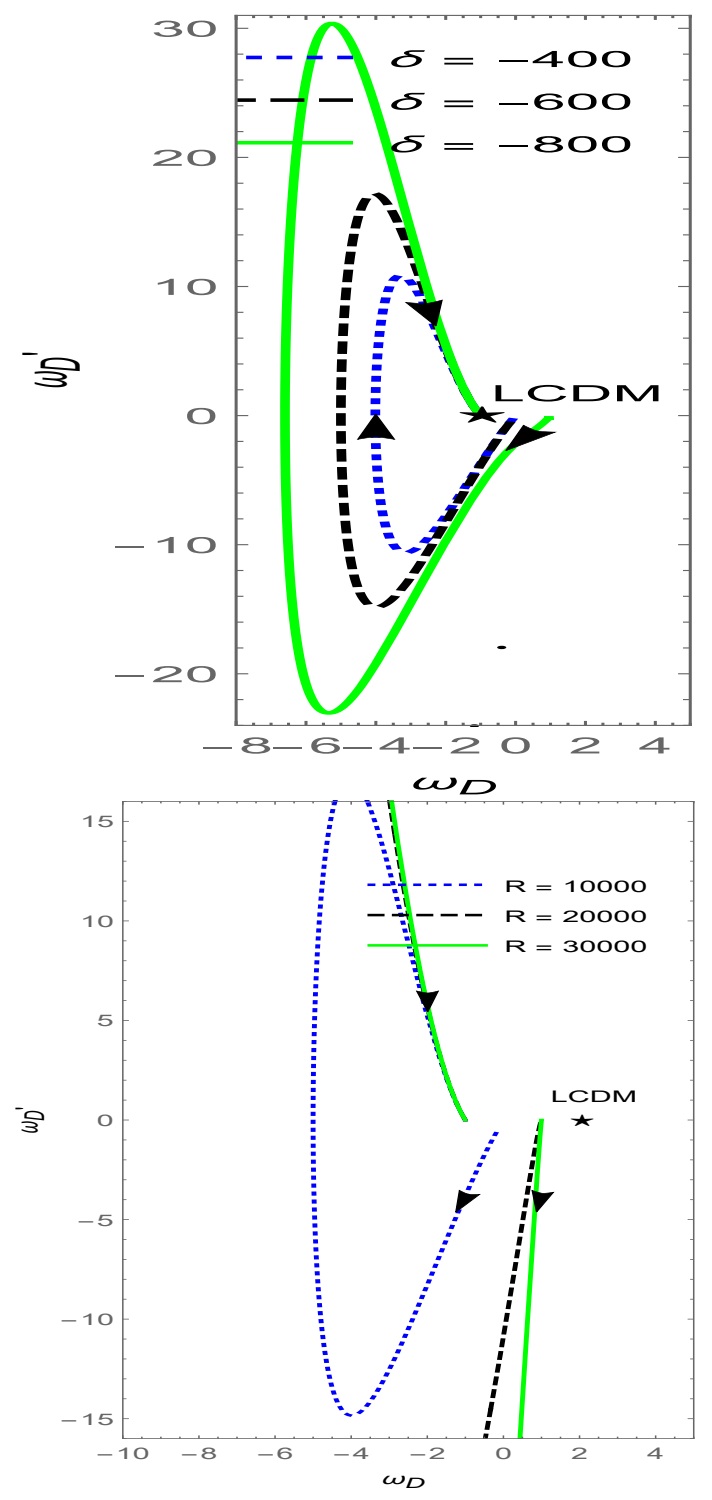

FIG. 8: The evolution trajectories in the $\omega_{D}-\omega_{D}^{\prime}$ plane of the SMHDE model, for non- interacting SMHDE with Hbbble radius as the IR cutoff. Here, $H(z=0)=67, \Omega_{m}(z=0)=$ $0.26, R=10000$ and different values of $\delta$ (upper panel) and $H(z=0)=67, \Omega_{m}(z=0)=0.26, \delta=-600$ and different values of $\mathrm{R}$ (below panel).

\section{The $\omega_{D}-\omega_{D}^{\prime}$ analysis}

The sign of $\omega_{D}^{\prime}$ can be used in the thawing and freezing models [78] and $\omega_{D}$ is the equation of state parameter characterizing the dark energy model. Hence $\omega_{D}-\omega_{D}^{\prime}$ pair analysis has been used to differentiate the similar model behaviours 83 89. where $\omega_{D}^{\prime}=\frac{d \omega_{D}}{d \log a}$. We investigated the dynamical diagnosis $\omega_{D}-\omega_{D}^{\prime}$ for SMHDE model which is also utilized widely in the literature. In this dynamical analysis, the fixed point $\omega_{D}=-1, \omega_{D}^{\prime}=0$ represents to the standard $\Lambda \mathrm{CDM}$ in the $\omega_{D}-\omega_{D}^{\prime}$ diagram.

The evolutionary trajectories of $\omega_{D}^{\prime}$ and $\omega_{D}$ plane are shown in Fig. 8, for an isotropic and spatially homogeneous flat FRW Universe of SMHDE model by considering different values of $\delta$ (upper panel) and $R$ (below panel). It is clear from $\omega_{D}-\omega_{D}^{\prime}$ trajectories by considering different values of $\delta$ (upper panel) that for all values of $\delta \omega_{D} \geq-1$ always and it crosses the phantom divide line $\omega_{D}=-1$. It also depicts that currently SMHDE model lies in the thawing region $\left(\omega_{D}<0, \omega_{D}^{\prime}>0\right)$ as well as in freezing region $\left(\omega_{D} \geq-1\right)$ which means presently, cosmic expansion is accelerating.

\section{CONCLUSIONS}

The paper uses the Sharma-Mittal Holographic Dark Energy (SMHDE) model in flat FRW Universe by considering different Sharma-Mittal parameter $\delta$. This can be summarized as

- We studied the deviation of SMHDE model from $\Lambda \mathrm{CDM}$ regarding different values of Sharma-Mittal parameter $\delta$ by the use of the diagnostics of statefinder hierarchy and growth rate of structure. The statefinder hierarchy gives analytical expressions of $S_{3}^{(1)}, S_{3}^{(2)}, S_{4}^{(1)}$ and $S_{4}^{(2)}$, for SMHDE in as cosmological parameters. To check the growth rate of structure $\epsilon(z)$ has been calculated analytically for the SMHDE model. We tested the SMHDE model using $\left\{S_{3}^{(1)}, \epsilon\right\}$ diagnostics. We plotted the evolution curves of $S_{3}^{(1)}, S_{3}^{(2)}, S_{4}^{(1)}$ and $S_{4}^{(2)}$ with respect to cosmic time $z$ and $\epsilon(z)$. These evolutionary trajectories shows that the SMHDE model shows $\Lambda$ CDM behaviour at late time. We have plotted the evolutionary trajectories of $\left\{S_{3}^{(1)}, \epsilon\right\}$ plane which depicts that SMHDE model for all values of $\delta$ shows same deviation from $\Lambda$ CDM model.

- The various diagnostic methods for dark energy have been discussed. we have also examined the $\omega_{D}-\omega_{D}^{\prime}$ pair analysis for our SMHDE model in subsection $C$. These analysis are used to differentiate among various dark energy models. In the subsection 5.1 we investigated dynamical diagnosis $\omega_{D}-\omega_{D}^{\prime}$ for SMHDE model where the derivative with respect to $\log \mathrm{a}$ is denoted by prime notation. The evolutionary trajectories of $\omega_{D}-\omega_{D}^{\prime}$ shows that presently cosmic expansion is accelerating since our SMHDE model lies in the thawing region $\left(\omega_{D}<0, \omega_{D}^{\prime}>0\right)$. It also indicates that $\omega_{D}$ crosses the phantom divide line $\omega_{D}=-1$ and $\omega_{D} \geq-1$. 
We have used three diagnostic tools in this work such as $\omega_{D}-\omega_{D}^{\prime}$ pair, the growth rate of perturbations and statefinder hierarchy to diagnose the SMHDE model. Some other diagnostic tool like statefinder diagnostic can also be used to discriminate the SMHDE from $\Lambda$ CDM model.

We hope that in future high precision observations, for example, SNAP-type investigation can be equipped for deciding the cosmological param- eters exactly and consequently identify the correct cosmological model and closer to understand the properties of the SMHDE model.

\section{Acknowledgments}

The authors are thankful for valuable suggestions given by Dr. Prateek Pandey, GLA University, Mathura, India, in this research work.
[1] A. G. Riess et al. [Supernova Search Team], "Observational evidence from supernovae for an accelerating universe and a cosmological constant," Astron. J. 116 (1998) 1009. doi:10.1086/300499.

[2] S. Perlmutter et al. [Supernova Cosmology Project Collaboration], "Measurements of $\Omega$ and $\Lambda$ from 42 high redshift supernovae," Astrophys. J. 517 (1999) 565. doi:10.1086/307221 astro-ph/9812133.

[3] N. Aghanim et al., Planck 2018 results. VI. Cosmological parameters. preprint (2018), arXiv:1807.06209 [astroph.CO].

[4] M. Colless et al. [2DFGRS Collaboration], "The $2 \mathrm{dF}$ Galaxy Redshift Survey: Spectra and redshifts," Mon. Not. Roy. Astron. Soc. 328 (2001) 1039 doi:10.1046/j.1365-8711.2001.04902.x

[5] M. Tegmark et al. [SDSS Collaboration], "Cosmological parameters from SDSS and WMAP," Phys. Rev. D 69 (2004) 103501 doi:10.1103/PhysRevD.69.103501

[6] D. N. Spergel et al. [WMAP Collaboration], "First year Wilkinson Microwave Anisotropy Probe (WMAP) observations: Determination of cosmological parameters," Astrophys. J. Suppl. 148 (2003) 175 doi:10.1086/377226

[7] E. J. Copeland, M. Sami and S. Tsujikawa, "Dynamics of dark energy," Int. J. Mod. Phys. D 15 (2006) 1753 doi:10.1142/S021827180600942X hep-th/0603057.

[8] K. Bamba, S. Capozziello, S. Nojiri and S. D. Odintsov, "Dark energy cosmology: the equivalent description via different theoretical models and cosmography tests," Astrophys. Space Sci. 342 (2012) 155 doi:10.1007/s10509012-1181-8

[9] W. Yang, S. Pan, E. Di Valentino, R. C. Nunes, S. Vagnozzi and D. F. Mota, "Tale of stable interacting dark energy, observational signatures, and the $H_{0}$ tension," JCAP 1809 (2018) 019. doi:10.1088/14757516/2018/09/019 arXiv:1805.08252 [astro-ph.CO]].

[10] L. Amendola, S. Tsujikawa, "Dark energy: theory and observations." Cambridge University Press (2010).

[11] V. Sahni, A. Starobinsky, "The case for a positive cosmological $\Lambda$-term," Int. J. Mod. Phys. D 9(04) (2000) 373-443.

[12] P. J. E. Peebles and B. Ratra, "The Cosmological constant and dark energy," Rev. Mod. Phys. 75 (2003) 559. doi:10.1103/RevModPhys.75.559

[13] T. Padmanabhan, "Dark energy and gravity," Gen. Rel. Grav. 40 (2008) 529. doi:10.1007/s10714-007-0555-7

[14] S. M. Carroll, "The Cosmological constant," Living Rev. Rel. 4 (2001) 1. doi:10.12942/lrr-2001-1

[15] S. Weinberg, "The Cosmological Constant
Problem," Rev. Mod. Phys. $61 \quad$ (1989) 1. doi:10.1103/RevModPhys.61.1

[16] P. J. E. Peebles and B. Ratra, "Cosmology with a timevariable cosmological'constant," Int. J. Mod. Phys. A 325 (1988) L17-L20.

[17] M. S.Turner, "Making sense of the new cosmology". Int. J. Mod. Phys. A, 17 (2002) 180.

[18] R. R. Caldwell and M. Kamionkowski et al., "Phantom energy and cosmic doomsday," Phys. Rev. Lett. 91 (2003) 071301. doi:10.1103/PhysRevLett.91.071301

[19] T. Chiba, "Tracking K-essence," Phys. Rev. D 66 (2002) 063514. doi:10.1103/PhysRevD.66.063514

[20] C. Armendariz-Picon, V. F. Mukhanov and P. J. Steinhardt, "Essentials of $k$ essence," Phys. Rev. D 63 (2001) 103510 doi:10.1103/PhysRevD.63.103510 astro$\mathrm{ph} / 0006373$.

[21] M. Malquarti, E. J. Copeland, A. R. Liddle and M. Trodden, "A New view of $k$-essence," Phys. Rev. D 67 (2003) 123503 doi:10.1103/PhysRevD.67.123503 astro$\mathrm{ph} / 0302279$.

[22] A. Sen, "Universality of the tachyon potential," J. High Energy Phys. 1999(12)(2000) 027.

[23] A. Y. Kamenshchik, U. Moschella et al., "An Alternative to quintessence," Phys. Lett. B 511 (2001) 265. doi:10.1016/S0370-2693(01)00571-8

[24] C.H. Brans and R.H. Dicke, "Mach's principle and a relativistic theory of gravitation," Phys. Rev. D 124(3) (1961) 925.

[25] A. De Felice and S. Tsujikawa, " $f(R)$ theories," Living Rev. Rel. 13 (2010) 3 doi:10.12942/lrr-2010-3

[26] S. Nojiri, S. D. Odintsov and V. K. Oikonomou, "Modified Gravity Theories on a Nutshell: Inflation, Bounce and Late-time Evolution," Phys. Rept. 692 (2017) 1 doi:10.1016/j.physrep.2017.06.001

[27] S. Nojiri and S. D. Odintsov, "Unified cosmic history in modified gravity: from $\mathrm{F}(\mathrm{R})$ theory to Lorentz non-invariant models," Phys. Rept. 505 (2011) 59 doi:10.1016/j.physrep.2011.04.001

[28] S.Maity and P. Rudra, (2018). Gravitational Baryogenesis in Ho $\check{r}$ ava-Lifshitz gravity. arXiv preprint arXiv:1802.00313

[29] T. Harko, F. S. N. Lobo, S. Nojiri and S. D. Odintsov, " $f(R, T)$ gravity," Phys. Rev. D 84 (2011) 024020 doi:10.1103/PhysRevD.84.024020

[30] L. Susskind, "The World as a hologram," J. Math. Phys. 36 (1995) 6377 doi:10.1063/1.531249

[31] P. Horava and D. Minic, "Probable values of the cosmological constant in a holographic theory," Phys. Rev. 
Lett. 85 (2000) 1610 doi:10.1103/PhysRevLett.85.1610

[32] S. D. Thomas, "Holography stabilizes the vacuum energy," Phys. Rev. Lett. 89 (2002) 081301. doi:10.1103/PhysRevLett.89.081301

[33] S. D. H. Hsu, "Entropy bounds and dark energy," Phys. Lett. B 594 (2004) 13 doi:10.1016/j.physletb.2004.05.020

[34] M. Li, "A Model of holographic dark energy," Phys. Lett. B 603 (2004) 1 doi:10.1016/j.physletb.2004.10.014

[35] S. Wang, Y. Wang and M. Li, "Holographic Dark Energy," Phys. Rept. 696 (2017) 1 doi:10.1016/j.physrep.2017.06.003

[36] S. Nojiri and S. D. Odintsov, "Unifying phantom inflation with late-time acceleration: Scalar phantomnon-phantom transition model and generalized holographic dark energy," Gen. Rel. Grav. 38 (2006) 1285 doi:10.1007/s10714-006-0301-6

[37] A. Sheykhi, "Holographic Scalar Fields Models of Dark Energy," Phys. Rev. D 84 (2011) 107302 doi:10.1103/PhysRevD.84.107302

[38] S. Srivastava, U. K. Sharma and A. Pradhan, "New holographic dark energy in bianchi- III Universe with $k$ essence," New Astron. 68, 57 (2019).

[39] Y. Z. Ma, Y. Gong and X. Chen, "Features of holographic dark energy under the combined cosmological constraints," Eur. Phys. J. C 60 (2009) 303 doi:10.1140/epjc/s10052-009-0876-7

[40] R. G. Cai, "A Dark Energy Model Characterized by the Age of the Universe," Phys. Lett. B 657 (2007) 228 doi:10.1016/j.physletb.2007.09.061

[41] H. Wei and R. G. Cai, "A New Model of Agegraphic Dark Energy," Phys. Lett. B 660 (2008) 113 doi:10.1016/j.physletb.2007.12.030

[42] C. Gao and F. Wu et al., "A Holographic Dark Energy Model from Ricci Scalar Curvature," Phys. Rev. D 79 (2009) 043511. doi:10.1103/PhysRevD.79.043511

[43] C. Tsallis and L. J. L. Cirto, "Black hole thermodynamical entropy," Eur. Phys. J. C 73 (2013) 2487 doi:10.1140/epjc/s10052-013-2487-6

[44] C. Tsallis, "Possible Generalization of BoltzmannGibbs Statistics," J. Statist. Phys. 52 (1988) 479. doi:10.1007/BF01016429

[45] A.Rényi , in Proceedings of the 4th Berkely Symposium on Mathematics, Statistics and Probability (University California Press, Berkeley, CA, 1961) pp. 547561.

[46] B.D. Sharma and D.P. Mittal, "New non-additive measures of entropy for discrete probability distributions," J. Math. Sci.10 (1975) 28-40; B.D. Sharma, D.P. Mittal, J. Comb. Inf. Syst. Sci. 2 (1977) 122.

[47] H. Moradpour, S. A. Moosavi, I. P. Lobo, J. P. Morais Graa, A. Jawad and I. G. Salako, "Thermodynamic approach to holographic dark energy and the Rnyi entropy," Eur. Phys. J. C 78 (2018) no.10, 829 doi:10.1140/epjc/s10052-018-6309-8

[48] M. Tavayef, A. Sheykhi, K. Bamba and H. Moradpour, "Tsallis Holographic Dark Energy," Phys. Lett. B $\mathbf{7 8 1}$ (2018) 195 doi:10.1016/j.physletb.2018.04.001

[49] M. Abdollahi Zadeh, A. Sheykhi and H. Moradpour, "Tsallis Agegraphic Dark Energy Model," Mod. Phys. Lett. A 34 (2019) no.11, 1950086 doi:10.1142/S021773231950086X

[50] A. Sayahian Jahromi, S. A. Moosavi, H. Moradpour, J. P. Morais Graa, I. P. Lobo, I. G. Salako and A. Jawad, "Generalized entropy formalism and a new holographic dark energy model," Phys. Lett. B $\mathbf{7 8 0}$ (2018) 21 doi:10.1016/j.physletb.2018.02.052

[51] A. Jawad, K. Bamba, M. Younas, S. Qummer and S. Rani, "Tsallis, Rnyi and Sharma-Mittal Holographic Dark Energy Models in Loop Quantum Cosmology," Symmetry 10 (2018) no.11, 635. doi:10.3390/sym10110635

[52] S. Nojiri, S. D. Odintsov and E. N. Saridakis, "Modified cosmology from extended entropy with varying exponent," Eur. Phys. J. C 79 (2019) 242.

[53] Q. Huang, H. Huang, J. Chen, L. Zhang and F. Tu, "Stability analysis of a Tsallis holographic dark energy model," Class. Quant. Grav. 36, no. 17, (2019) 175001. S. Ghaffari, H. Moradpour, I. P. Lobo, J. P. Morais Graa and V. B. Bezerra, "Tsallis holographic dark energy in the BransDicke cosmology," Eur. Phys. J. C 78 (2018) no.9, 706 doi:10.1140/epjc/s10052-018-6198-x

[54] E. N. Saridakis, K. Bamba, R. Myrzakulov and F. K. Anagnostopoulos, "Holographic dark energy through Tsallis entropy," JCAP $\mathbf{1 8 1 2}$ (2018) 012 doi:10.1088/1475-7516/2018/12/012

[55] V. C. Dubey, S. Srivastava, U. K. Sharma and A. Pradhan, "Tsallis holographic dark energy in Bianchi-I Universe using hybrid expansion law with $k$-essence," Pramana 93 (2019) no.5, 78. doi:10.1007/s12043-019-1843-y

[56] E. Sadri, "Observational constraints on interacting Tsallis holographic dark energy model," Eur. Phys. J. C 79 (2019) no.9, 762 doi:10.1140/epjc/s10052-019-7263-9

[57] E. M. Barboza, Jr., R. d. C. Nunes, E. M. C. Abreu and J. Ananias Neto, "Dark energy models through nonextensive Tsallis statistics," Physica A 436 (2015) 301 doi:10.1016/j.physa.2015.05.002

[58] Golanbari, T., Saaidi, K., Karimi, P. (2020). Renyi entropy and the holographic dark energy in flat space time. arXiv preprint arXiv:2002.04097.

[59] Sharma, U. K., Dubey, V. C. (2020). Interacting Rényi holographic dark energy with parametrization on the interaction term. arXiv preprint arXiv:2001.02368

[60] S. Ghaffari, A. H. Ziaie, V. B. Bezerra and H. Moradpour, "Inflation in the Rnyi cosmology," Mod. Phys. Lett. A 35 (2019) no.01, 1950341 doi:10.1142/S0217732319503413

[61] V. C. Dubey, et al. "Tsallis holographic dark energy Models in axially symmetric space time." Int. J. Geom. Methods Mod. Phys. 171 (2020) 2050011.

[62] Y. Aditya, S. Mandal, P. K. Sahoo and D. R. K. Reddy, "Observational constraint on interacting Tsallis holographic dark energy in logarithmic BransDicke theory," Eur. Phys. J. C 7912, 1020 (2019).

[63] A. Iqbal and A. Jawad, "Tsallis, Renyi and SharmaMittal holographic dark energy models in DGP brane-world," Phys. Dark Univ. 26 (2019) 100349. doi:10.1016/j.dark.2019.100349

[64] M. Arabsalmani and V. Sahni, "The Statefinder hierarchy: An extended null diagnostic for concordance cosmology," Phys. Rev. D 83 (2011) 043501. doi:10.1103/PhysRevD.83.043501

[65] J. F. Zhang and J. L. Cui et al., "Diagnosing holographic dark energy models with statefinder hierarchy," Eur. Phys. J. C $\mathbf{7 4}$ (2014) no.10, 3100. doi:10.1140/epjc/s10052-014-3100-3

[66] V. Acquaviva and A. Hajian et al., "Next Generation Redshift Surveys and the Origin of Cosmic Acceleration," Phys. Rev. D 78 (2008) 043514. doi:10.1103/PhysRevD.78.043514

[67] V. Acquaviva and E. Gawiser, "How to Falsify 
the GR+LambdaCDM Model with Galaxy Redshift Surveys," Phys. Rev. D 82 (2010) 082001. doi:10.1103/PhysRevD.82.082001

[68] R. Myrzakulov and M. Shahalam, "Statefinder hierarchy of bimetric and galileon models for concordance cosmology," JCAP 1310 (2013) 047. doi:10.1088/1475$7516 / 2013 / 10 / 047$

[69] J. Li and R. Yang et al., "Discriminating dark energy models by using the statefinder hierarchy and the growth rate of matter perturbations," JCAP 1412 (2014) 043. doi:10.1088/1475-7516/2014/12/043

[70] Y. Hu and M. Li et al., "Impacts of different SNLS3 lightcurve fitters on cosmological consequences of interacting dark energy models," Astron. Astrophys. 592 (2016) A101. doi:10.1051/0004-6361/201526946

[71] A. Mukherjee, N. Paul and H. K. Jassal, "Constraining the dark energy statefinder hierarchy in a kinematic approach," JCAP 1901 (2019) 005 doi:10.1088/14757516/2019/01/005

[72] Cui, J. L., et al., " A closer look at interacting dark energy with statefinder hierarchy and growth rate of structure". JCAP 2015(09) (2015) 024.

[73] Zhou, L., Wang, S., "Diagnosing $\Lambda$ HDE model with statefinder hierarchy and fractional growth parameter". China Phys. Mech. Astron. 59(7) (2016) 670411.

[74] Majumdar, A., Chattopadhyay, S., "A study of modified holographic Ricci dark energy in the framework of $\mathrm{f}(\mathrm{T})$ modified gravity and its statefinder hierarchy". Can. J. Phys. 97(5) ( 2018) 477-486.

[75] Zhao, Z., Wang, S., "Diagnosing holographic type dark energy models with the Statefinder hierarchy, composite null diagnostic and $\omega_{D}-\omega_{D}^{\prime}$ pair". China Phys. Mech. Astron. 61(3) (2018) 039811.

[76] Yu, F., et al., "Statefinder hierarchy exploration of the extended Ricci dark energy". Eur. Phys. J. C $\mathbf{7 5}(6)(2015) 274$.

[77] Srivastava, Vandna, and Umesh Kumar Sharma. "Statefinder hierarchy for Tsallis holographic dark energy." New Astronomy (2020): 101380.

[78] R. R. Caldwell and E. V. Linder, "The Limits of quintessence," Phys. Rev. Lett. 95 (2005) 141301.
doi:10.1103/PhysRevLett.95.141301

[79] A. G. Cohen, D. B. Kaplan and A. E. Nelson, "Effective field theory, black holes, and the cosmological constant," Phys. Rev. Lett. 82 (1999) 4971 doi:10.1103/PhysRevLett.82.4971

[80] V. Sahni and A. Shafieloo et al., "Two new diagnostics of dark energy," Phys. Rev. D 78 (2008) 103502. doi:10.1103/PhysRevD.78.103502

[81] L. M. Wang and P. J. Steinhardt, "Cluster abundance constraints on quintessence models," Astrophys. J. 508 (1998) 483. doi:10.1086/306436

[82] E. V. Linder, "Cosmic growth history and expansion history," Phys. Rev. D 72 (2005) 043529. doi:10.1103/PhysRevD.72.043529

[83] M. Malekjani and A. Khodam-Mohammadi, "Agegraphic Dark Energy Model in Non-Flat Universe: Statefinder Diagnostic and $w-w^{\prime}$ Analysis," Int. J. Mod. Phys. D 19 (2010) 1857. doi:10.1142/S0218271810018086

[84] A. Khodam-Mohammadi and M. Malekjani, "Cosmic Behavior, Statefinder Diagnostic and $w-w^{\prime}$ Analysis for Interacting NADE model in Non-flat Universe," Astrophys. Space Sci. 331 (2011) 265. doi:10.1007/s10509-010-0422$\mathrm{y}$

[85] U. K. Sharma and A. Pradhan, "Diagnosing Tsallis holographic dark energy models with statefinder and $\omega_{D}-\omega_{D}^{\prime}$ pair," Phys. Lett. A 34(13) (2019) 1950101.

[86] G. Varshney and U. K. Sharma et al., "Statefinder diagnosis for interacting Tsallis holographic dark energy models with $\omega-\omega^{\prime}$ pair," New Astron. 70 (2019) 36. doi:10.1016/j.newast.2019.02.004

[87] N. Zhang and Y. B. Wu et al., "Diagnosing the interacting Tsallis Holographic Dark Energy models," arXiv preprint (2019). arXiv:1905.04299

[88] V. C. Dubey and U. K. Sharma et al., "Tsallis holographic model of dark energy: Cosmic behaviour, statefinder analysis and $\omega_{D}-\omega_{D}^{\prime}$ pair in the non-flat universe," (2019). arXiv preprint arXiv:1905.02449.

[89] S. Srivastava, V. C. Dubey, U. K. sharma, "Statefinder diagnosis for Tsallis agegraphic dark energy model with $\omega_{D}-\omega_{D}^{\prime}$ pair," Int. J. Mod. Phys. A 35, 2050027 (2020). 